\title{
The Video-Urodynamic and Electrophysiological Characteristics in Patients With Traumatic Spinal Cord Injury
}

\author{
Zhaoxia Wang ${ }^{1}$, Han Deng ${ }^{2,3}$, Xing $\mathrm{Li}^{2,3}$, Limin $\mathrm{Liao}^{2,3}$ \\ ${ }^{1}$ Department of Rehabilitation Medicine, Beijing Tiantan Hospital, Capital Medical University, Beijing, China \\ ${ }^{2}$ Department of Urology, China Rehabilitation Research Centre, Rehabilitation School of Capital Medical University, Beijing, China \\ ${ }^{3}$ Center of Neural Injury and Repair, Beijing Institute for Brain Disorders, Beijing, China
}

\begin{abstract}
Purpose: To investigate the video-urodynamic and pelvic floor electrophysiological characteristics in patients with traumatic spinal cord injury (SCI).

Methods: This retrospective reviewed the clinical records, urodynamic and pelvic floor electrophysiological data of $647 \mathrm{pa}-$ tients with traumatic SCI and out of spinal shock. Patients were classified based on American Spinal Injury Association (ASIA) Impairment Scale and urodynamic findings.

Results: Of the 647 patients, detrusor overactivity (DO) with or without detrusor sphincter dyssynergia (DSD) was found in $79.5 \%, 61 \%, 35.2 \%, 35 \%$, and $19.2 \%$ of patients with cervical, thoracic (T1-9), thoracic (T10-12), lumbar, and conical cauda injury, respectively. Other patients manifested detrusor areflexia (DA). Patients with DO and/or DSD had a longer duration of SCI at each injury level than patients with DA. In suprasacral injury patients with DA, 63.0\% (58 of 92) had a normal bulbocavernosus reflex (BCR) response. Compared with patients without bladder sensation (BS), bladder capacity during urine leakage was far higher in those with BS. The manifestation of BCR and somatosensory-evoked potential (SEP) was associated with the level of injury.

Conclusions: This study showed a significant correlation between the level of SCI and video-urodynamic findings, but clinical examination cannot by predict bladder function; urodynamic testing is also necessary. In addition, the role of BCR and SEP for guiding bladder management is limited. Moreover, BS is important for urinary control in patients with traumatic SCI.
\end{abstract}

Keywords: Traumatic spinal cord injury; Video-urodynamic findings; Pelvic floor electrophysiological data

- Fund Support: This study was funded by the National Natural Science Foundation of China (No.81570688).

- Research Ethics: This study was approved by the Institutional Review Board of China Rehabilitation Research Centre (No.2017-050-1).

- Conflict of Interest: No potential conflict of interest relevant to this article was reported.

\section{INTRODUCTION}

Urodynamic findings are reported to be significantly associated with somatic neurologic findings in patients with spinal cord injury (SCI), but the correlation is not exact [1-4]. After a period of spinal shock, suprasacral injury theoretically results in detrusor overactivity (DO) and/or detrusor sphincter dyssynergia
(DSD). In contrast, injury to or below the sacral cord, including conus and cauda injuries, should lead to detrusor areflexia (DA) $[3,5]$. However, previous studies have shown that many patients with SCI have varied urodynamic findings that cannot easily predict bladder and sphincter behavior only with clinical neurological examination [1-4]. Bulbocavernosus reflex (BCR) and somatosensory-evoked potential (SEP) also have some relation-
Corresponding author: Limin Liao (iD https://orcid.org/0000-0002-7092-6576 Department of Urology, China Rehabilitation Research Center, No 10. Jiaomen Beilu, Fengtai District, Beijing 100068, China

Email:1mliao@263.net

Submitted: October 6, 2020 / Accepted after revision: December 23, 2020
This is an Open Access article distributed under the terms of the Creative Commons Attribution Non-Commercial License (https://creativecommons.org/licenses/by-nc/4.0/) which permits unrestricted non-commercial use, distribution, and reproduction in any medium, provided the original work is properly cited. 
ship with clinical findings in SCI patients [6], but neither these data can accurately predict DO/DSD nor DA [7].

However, the correlation of somatic neurologic findings, urodynamic findings, and electrophysiological data has not been fully researched in patients with SCI, especially the relationship of bladder sensation (BS) and somatic neurologic findings. This study combined American Spinal Injury Association (ASIA) Impairment Scale, video-urodynamic studies, and pelvic floor electrophysiological tests to find the possible connections among them in 647 patients with traumatic SCI.

\section{MATERIALS AND METHODS}

\section{Patients}

With Institutional Review Board of China Rehabilitation Research Centre (CRRC) approval, we retrospectively reviewed 647 patients (536 males and 111 females) with posttraumatic SCI of the CRRC from February 2012 to July 2018. All patients recovered from spinal shock but still required intermittent or continuous catheter drainage to empty the bladder. The mean patient age was $34.7 \pm 13.8$ years, and the mean injury history was $7.2 \pm 8.4$ months. All patients received somatic neurological examination and were categorized according to the ASIA Impairment Scale [8]. Video-urodynamic evaluation and pelvic floor electrophysiological examination were performed during the same week. None of patients were diagnosed with symptomatic urinary tract infection, kidney stones, or other lower urinary dysfunction before injury.

\section{Video-Urodynamic Evaluation}

All patients underwent video-urodynamic evaluation to measure BS, bladder, and sphincter behavior. All urodynamic measurement was performed according to Good Urodynamic Practices [9]. Symptoms of DO were strictly monitored, especially in patients with cervical and high thoracic lesions. The condition of the urethra and bladder neck was observed by x-ray when DO occurred (Fig. 1).
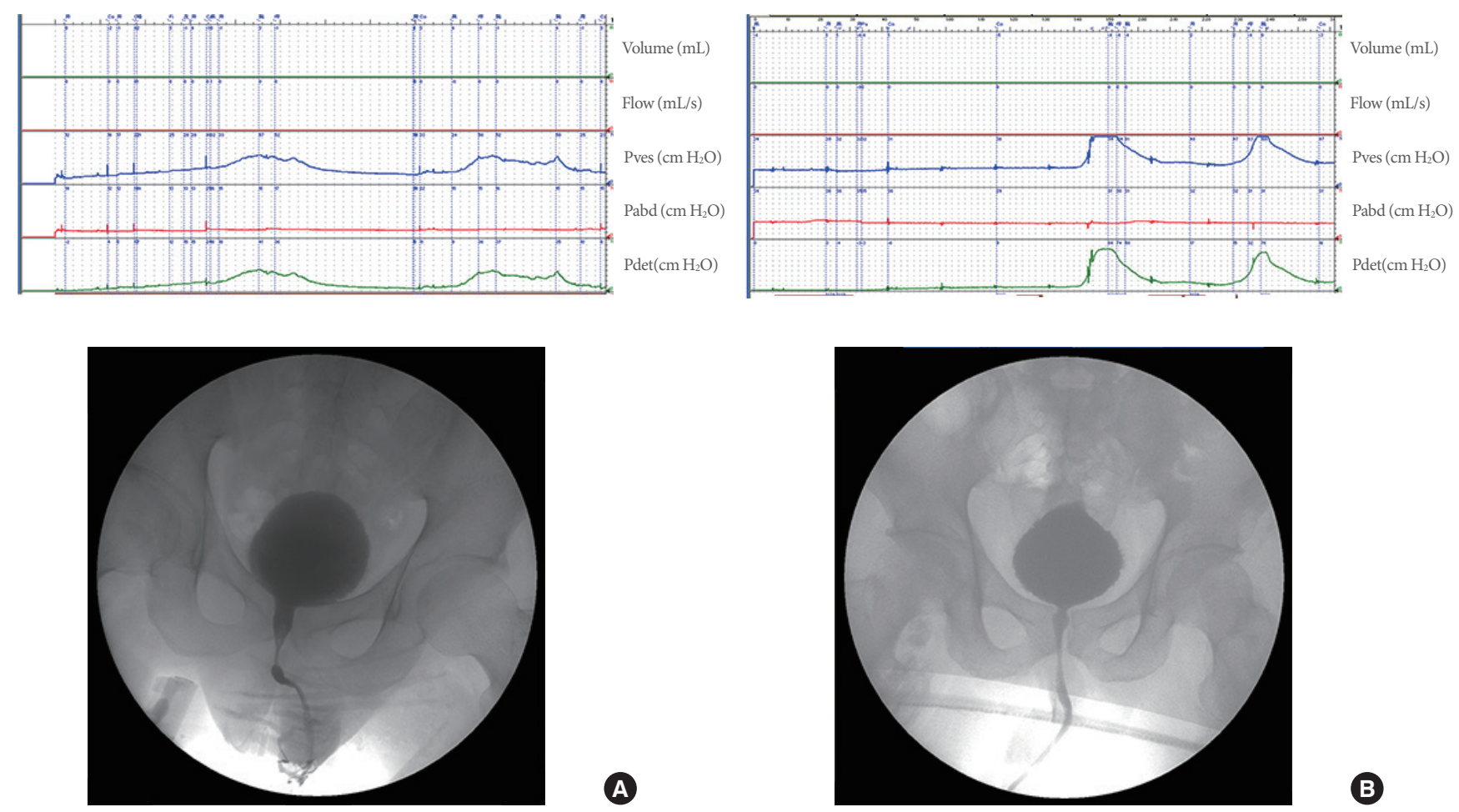

A

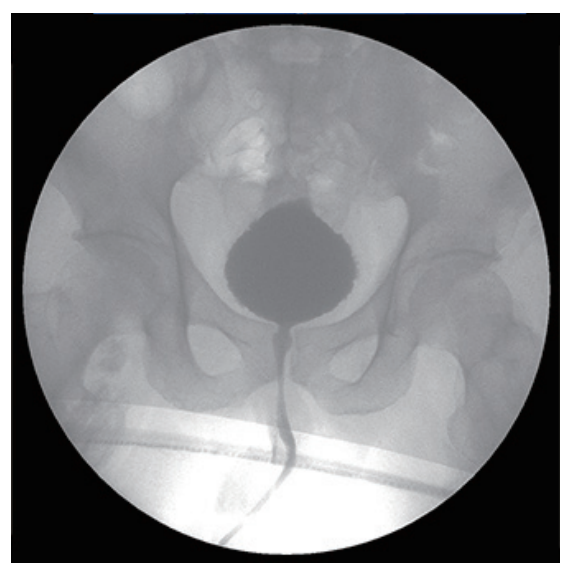

B

Fig. 1. (A) Video-urodynamic study in patient with complete thoracic 8 injury and detrusor sphincter dyssynergia. The external urethral sphincter remains narrow during detrusor hyperreflexia contractions. (B) Video-urodynamic study in patient with incomplete cervical 4 spinal cord injury, detrusor sphincter dyssynergia, and detrusor bladder neck dyssynergia. The bladder neck and external urethral sphincter remain narrow during detrusor hyperreflexia contractions. 


\section{Pelvic Floor Electrophysiological Examination}

The electrophysiological examination consisted of BCR and pudendal SEP, motor nerve conduction (MNC), sympathetic skin response, anal sphincter electrophysiology (EMG). Because many patients refused some examinations, especially MNC and EMG, only BCR and pudendal SEP were performed in all patients. Thus, we analyzed only the BCR and SEP data in this study.

\section{Standard Definitions}

DO is a urodynamic observation characterized by involuntary detrusor contractions during the filling phase that may be spontaneous or provoked [10]. DSD is a detrusor contraction concurrent with an involuntary contraction of the urethral and/ or periurethral striated muscle. In this study, we defined DSD as a video-urodynamic observation of partial or complete closure of the urethra during detrusor contraction (Fig. 1A). Using a video-urodynamic study, detrusor bladder neck dyssynergia (DBND) can be clearly demonstrated as a narrow bladder neck during voluntary or involuntary voiding (Fig. 1B). In this study, we measured the DBND only in patients with DO. The first sensation of bladder filling was patients' sensation of bladder filling during cystometry [10]. BS was categorized as absent or preserved in this study. Low bladder compliance (BC) was defined as $<20 \mathrm{~mL} / \mathrm{cm} \mathrm{H}_{2} \mathrm{O}$.

\section{Statistical Analysis}

A chi-square test was used to determine the association of the level of injury with video-urodynamic findings, BCR, and SEP and the association of the degree of injury and BS. A paired Student $\mathrm{t}$-test was used to compare the latency and amplitude of BCR in patients with injuries of different degrees, the latency and amplitude in patients with and without SEP, the leak point pressure and the bladder capacity of urine leakage in DO patients with and without SEP, and the leak point pressure and bladder capacity of urine leakage in DO patients with and without BS. Statistical analysis was performed using IBM SPSS Statistics ver. 19.0 (IBM Co., Armonk, NY, USA). P $<0.05$ was considered statistically significant.

\section{RESULTS}

Of the 647 patients, 184 (28.4\%) had cervical injury, 325 (50.2\%) had thoracic injury, 38 (6\%) had lumbar injury, and 99 (15.3\%) had conical and/or cauda injury, according to ASIA. In patients

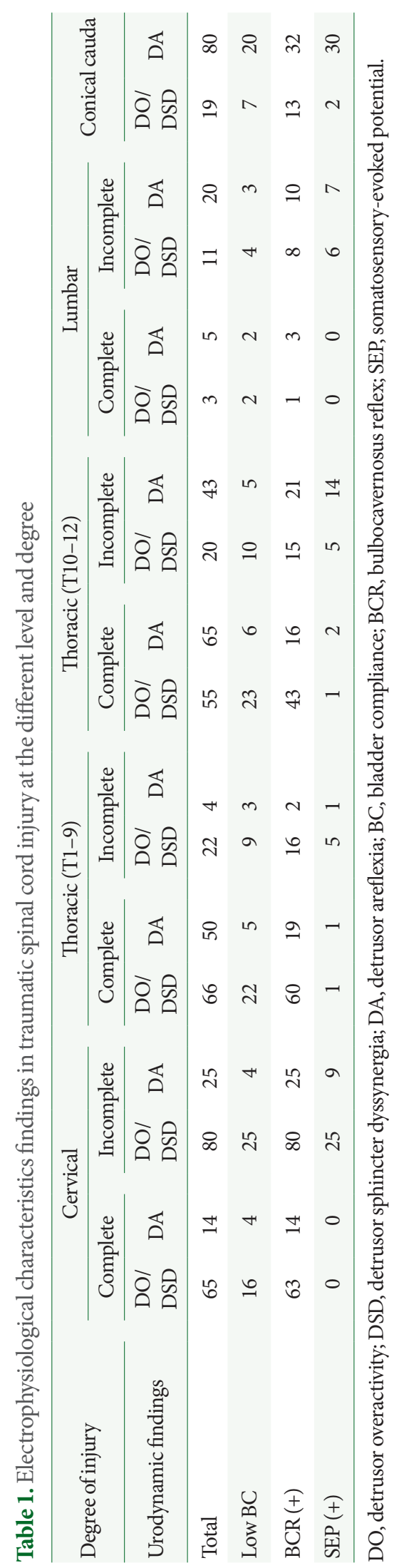


with thoracic injury, 142 (21.9\%) had T1-9 injury, and 183 (28.3\%) had T10-12 injury. In patients with suprasacral injury, 323 (49.9\%) had complete injury, and 225 (34.8\%) had incomplete injury, according to ASIA Impairment Scale (Table 1).

There was no association between the degree of suprasacral injury and the urodynamic findings $(\mathrm{P}=0.89>0.05)$ (Fig. $2 \mathrm{~A}$ ). Fig. $2 \mathrm{~B}$ shows the association of level of injury and urodynamic findings $(\mathrm{P}<0.01)$. Of the 323 patients with complete suprasacral injuries, 189 (58.5\%) had DO/DSD, and 134 (41.5\%) had DA. In patients with DA, 39\% (54 of 134) had a normal BCR response. Of the 225 patients with incomplete suprasacral injuries, 133 (59\%) had DO/DSD, and 92 (41\%) had DA. In patients with DA, 63\% (58 of 92) had a normal BCR response. In addition, in patients with conical and/or cauda injury, 19.2\% had DO/DSD, and 80.8\% had DA. In patients with DA, 40\% (32 of 80) had a normal BCR response (Supplementary Material).

DBND was associated with the level rather than the degree of injury (Fig. 2C, D). DBND was most common in patients with cervical or high thoracic SCI. Of the 57 patients with DBND, 53 had DSD.

The association between duration of injury of DO/DSD and
DA in patients with traumatic SCI patients is shown in Fig. 3. Patients with DO/DSD had a significantly longer duration of injury than those with DA at each injury level.

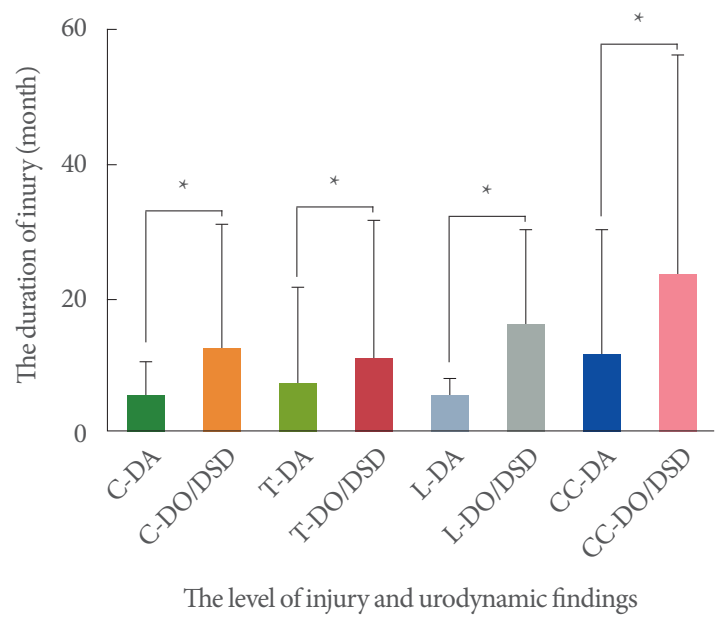

Fig. 3. Comparison of the duration of injury and different urodynamic findings at each level. C, cervical; T, thoracic; L, lumbar; CC, conical and/or cauda injury; DO, detrusor overactivity; DSD, detrusor sphincter dyssynergia; DA, detrusor areflexia. ${ }^{*} \mathrm{P}<0.05$, significant difference.

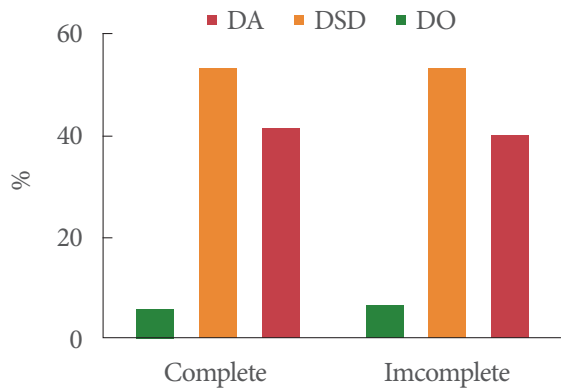

The degree of injury

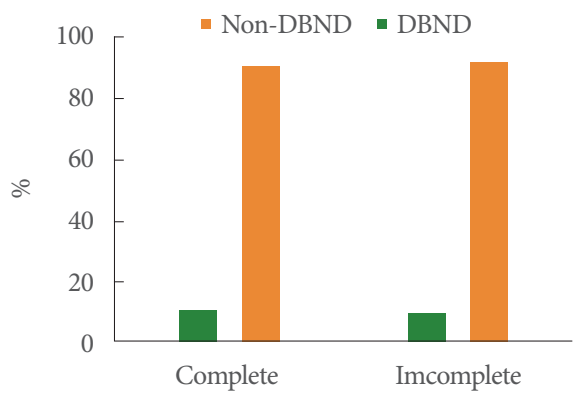

The degree of injury
A
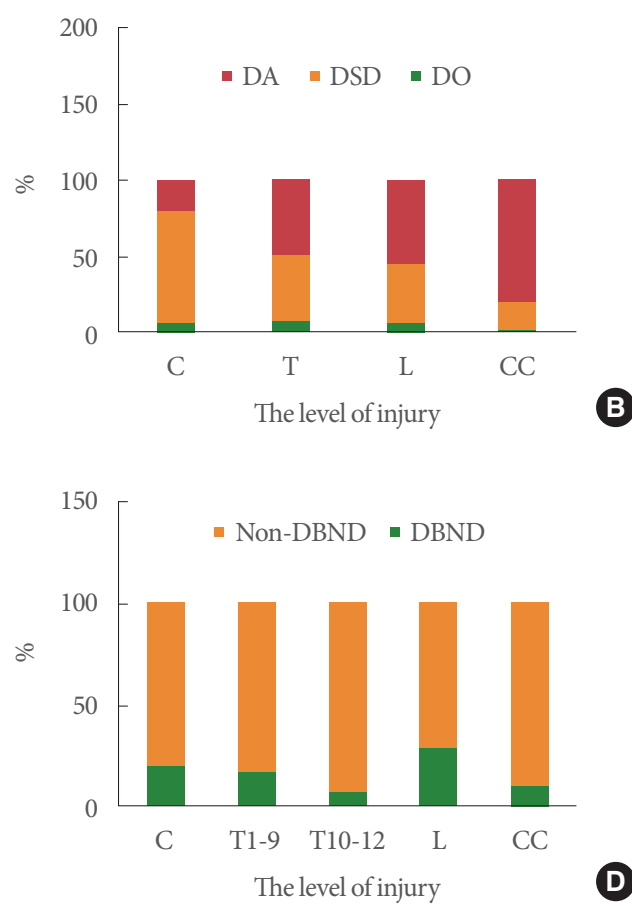

Fig. 2. Urodynamic findings at different degree $(A ; P=0.95>0.05)$ and level $(B ; P<0.001)$ of spinal cord injury. Percentage of detrusor bladder neck dyssynergia at different degree $(\mathrm{C} ; \mathrm{P}=0.81>0.05)$ and level $(\mathrm{D} ; \mathrm{P}=0.0004)$ of spinal cord injury. C. cervical; $\mathrm{T}$, thoracic; L, lumbar; CC, conical and/or cauda injury; DO, detrusor overactivity; DSD, detrusor sphincter dyssynergia; DA, detrusor areflexia; DBND, detrusor bladder neck dyssynergia. 

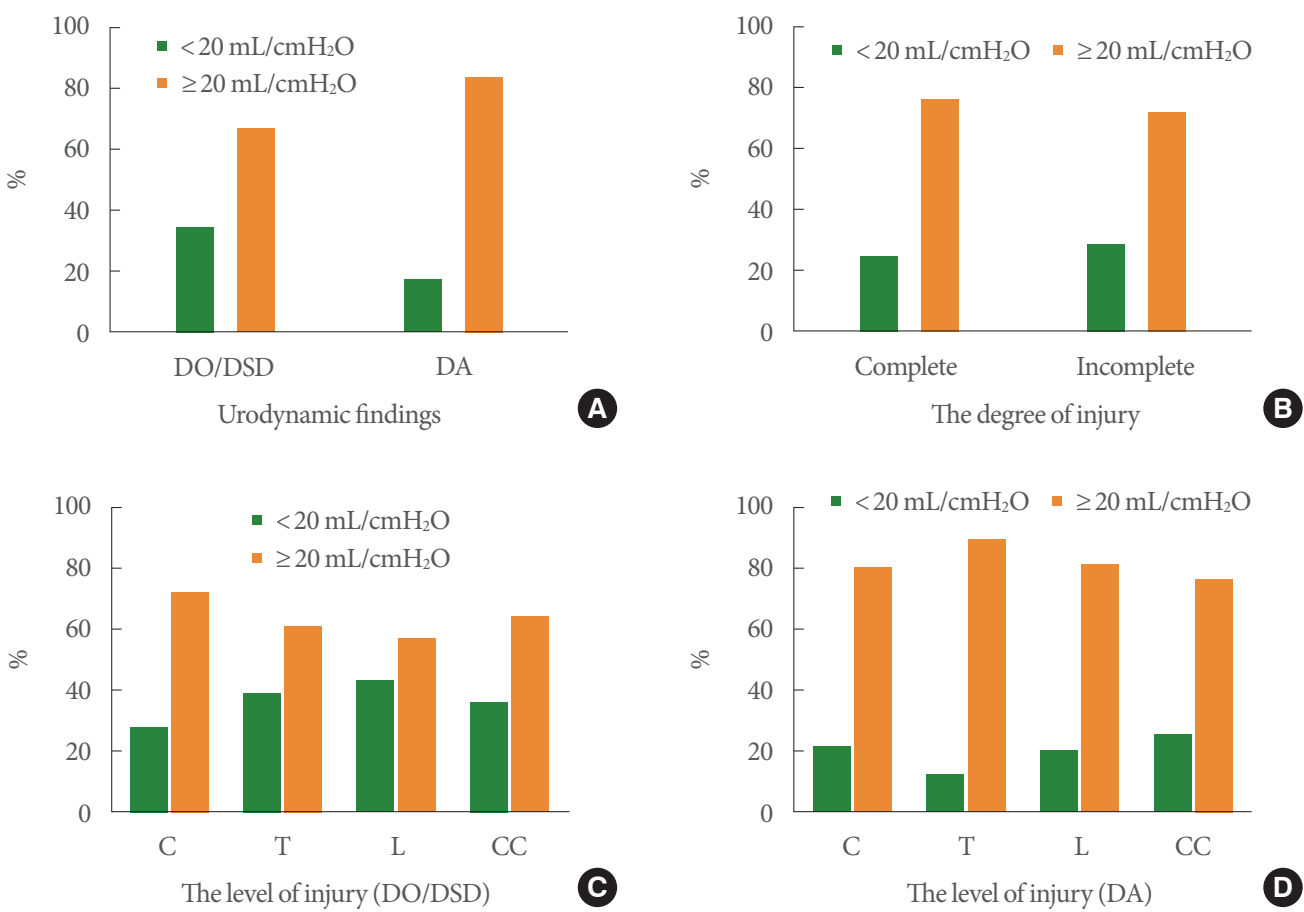

Fig. 4. Percentage of patients with low bladder compliance as determined by urodynamic findings (detrusor reflexes; $A, P=$ $0.0058<0.01$ ), degree of injury ( $\mathrm{B}, \mathrm{P}=0.16<0.05)$, and level of injury ( $\mathrm{C}, \mathrm{DO} / \mathrm{DSD}$ group, $\mathrm{P}=0.13>0.05 ; \mathrm{D}, \mathrm{DA}$ group $\mathrm{P}=0.52>0.05)$. $\mathrm{C}$, cervical; T, thoracic; L, lumbar; CC, conical and/or cauda injury; DO, detrusor overactivity; DSD, detrusor sphincter dyssynergia; DA, detrusor areflexia.
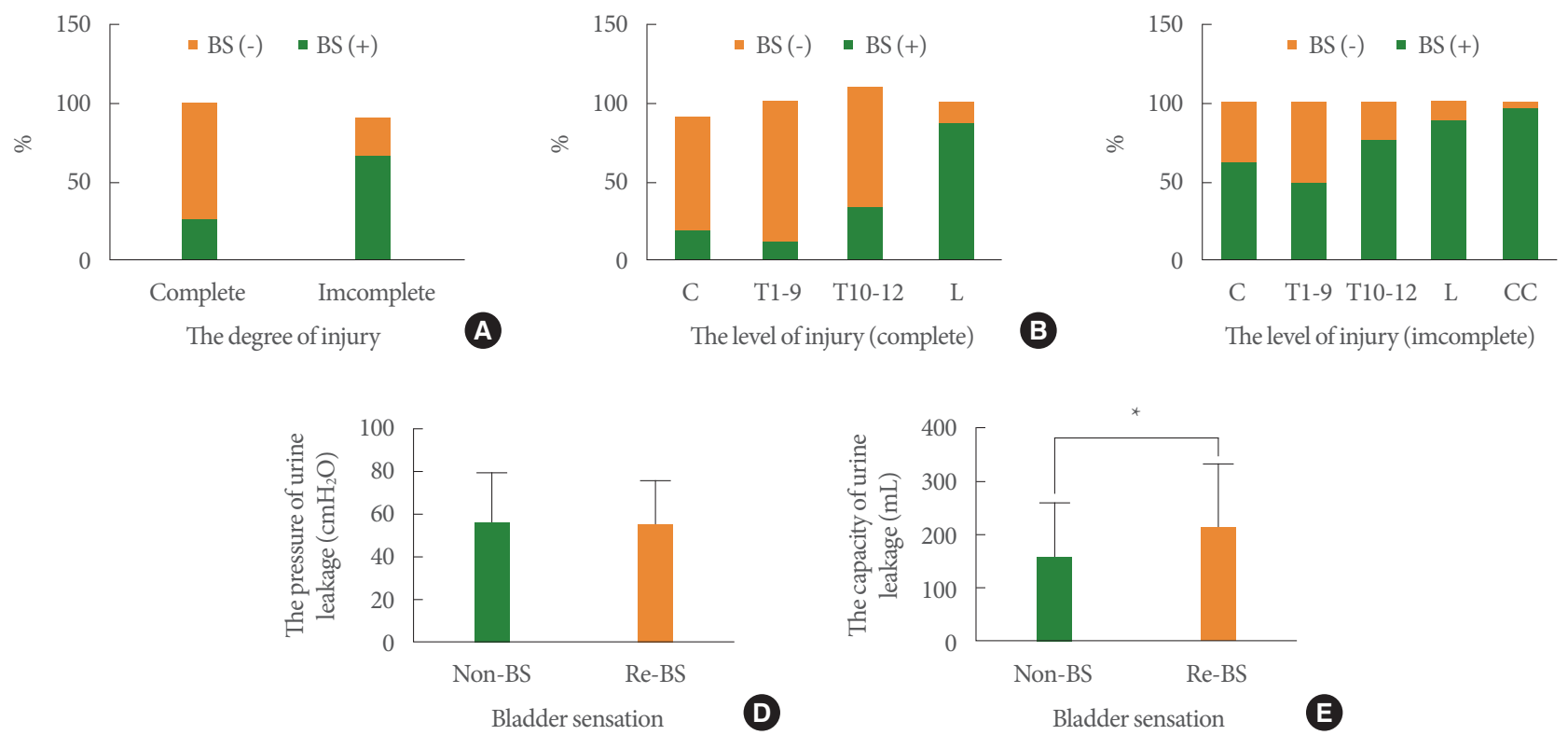

Fig. 5. Percentage of patients with bladder sensation of different degrees $(A, P<0.0001)$ and levels (B, complete group, $P<0.0001$; $C$, incomplete group, $\mathrm{P}<0.0001)$. Comparison of pressure of urine leakage $(\mathrm{D}, \mathrm{P}=0.56>0.05)$ and bladder capacity during urine leakage in patients with detrusor overactivity/detrusor sphincter dyssynergia, with and without bladder sensation, respectively $(\mathrm{E}, \mathrm{P}=$ $0.0003<0.05)$. C, cervical; $T$, thoracic; L, lumbar; CC, conical and/or cauda injury; BS, bladder sensation. ${ }^{*} \mathrm{P}<0.05$, significant difference. 

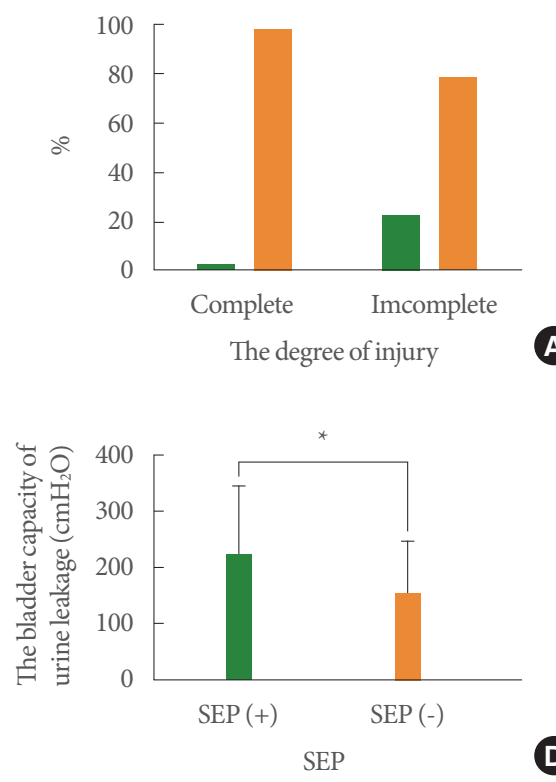

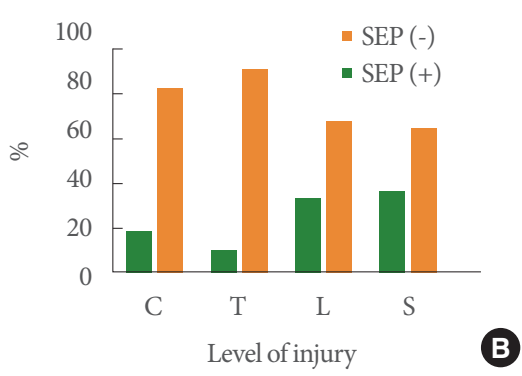

B

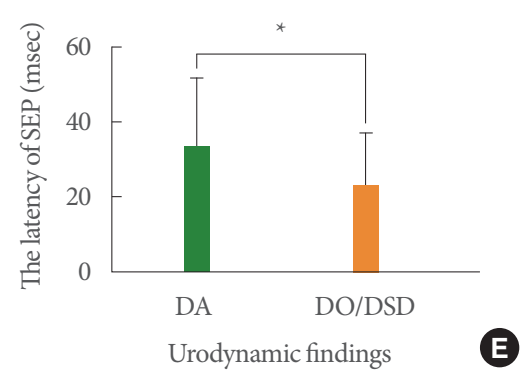

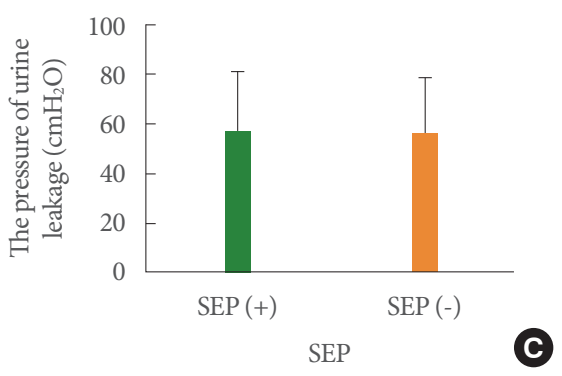

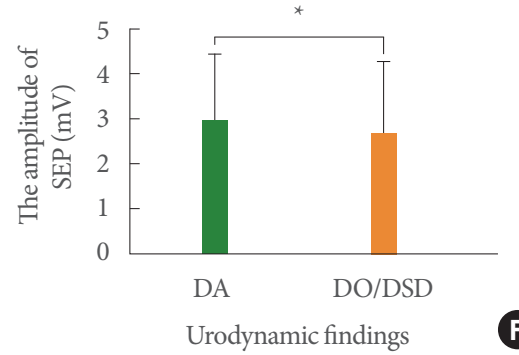

Fig. 6. Percentage of patients with positive pudendal SEP of different degrees $(A, P<0.0001)$ and levels $(B, P<0.0001)$. Comparison of pressure of urine leakage $(\mathrm{C}, \mathrm{P}=0.44>0.05)$ and bladder capacity during urine leakage in patients with detrusor overactivity/detrusor sphincter dyssynergia, with and without pudendal SEP, respectively $(\mathrm{D}, \mathrm{P}=0.037<0.05)$. Comparison of latency $(\mathrm{E}, \mathrm{P}<0.05)$ and amplitude (F) of pudendal SEP in patients with positive pudendal SEP and different urodynamic findings. C, cervical; T, thoracic; L, lumbar; S, sacral; DO, detrusor overactivity; DSD, detrusor sphincter dyssynergia; DA, detrusor areflexia; SEP, pudendal somatosensoryevoked potential. ${ }^{*} \mathrm{P}<0.05$, significant difference.
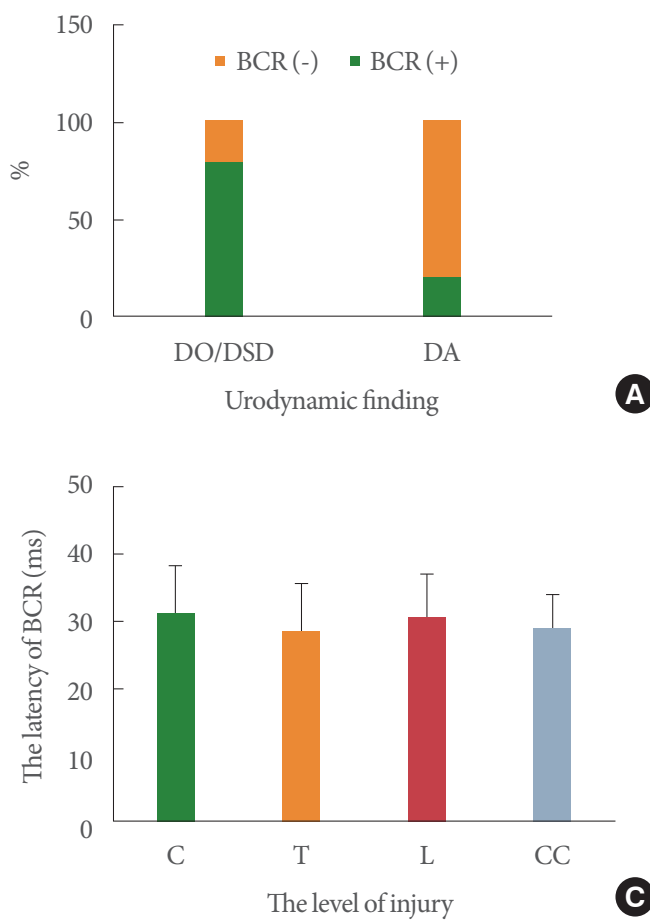
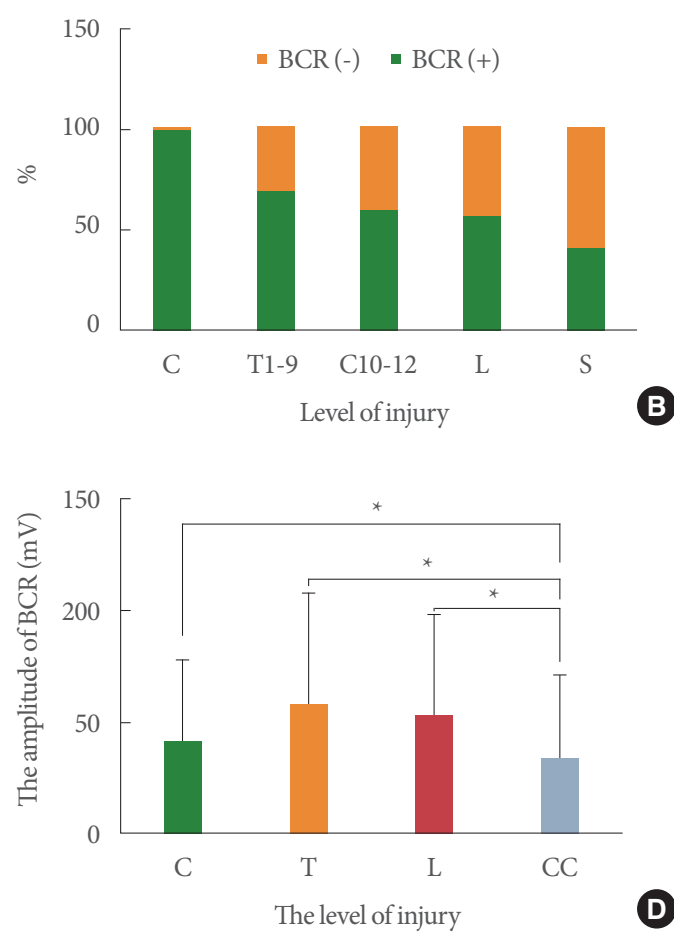

Fig. 7. Percentage of patients with positive $B C R$ and urodynamic findings $(A, P<0.0001)$ and level of injury $(B, P<0.0001)$. Comparison of latency (C) and amplitude (D) of BCR in patients with positive BCR at each level. C, cervical; T, thoracic; L, lumbar; S, sacral; CC, conical and/or cauda injury; BCR, bulbocavernosus reflex; DO, detrusor overactivity; DSD, detrusor sphincter dyssynergia; DA, detrusor areflexia. ${ }^{\star} \mathrm{P}<0.05$, significant difference from the conical and/or cauda group. 
Fig. 4A shows that $\mathrm{BC}$ was associated with urodynamic findings (detrusor reflexes, $\mathrm{P}<0.01$ ) rather than level of SCI injury. As shown in Fig. 4, 117 patients (34\%) with DO/DSD and 52 (17\%) with DA presented with low BC. In patients with suprasacral and conical and/or cauda injury, 76 (24\%) and 27 (27\%), respectively, had low $\mathrm{BC}$.

Fig. 5A-C shows the relationship of level and degree of injury and the reserved $\mathrm{BS} /$ urge to empty $(\mathrm{P}<0.05)$. BS was observed in $26 \%$ and $67 \%$ of patients with complete and incomplete suprasacral injury, respectively. In patients with either complete or incomplete injury, BS seemed more likely to be reserved in lumbar injury or 10-12 thoracic injury than those with cervical injury or $1-9$ thoracic injury $(\mathrm{P}<0.05)$. In both the complete and incomplete injury groups, there was no significant difference about reserved BS cervical injury group and those with a 1-9 thoracic injury group.

In patients with $\mathrm{DO} / \mathrm{DSD}$, pressure of urine leakage had no association with the BS (Fig. 5D) $(\mathrm{P}=0.56)$. However, there was significant difference in bladder capacity in leakage in patients with and without BS.

Fig. $6 \mathrm{~A}$ shows the association between the level of incomplete injury and the frequency of SEP. The frequency of SEP in patients with a lumbar injury or conical and/or cauda injury was significantly higher than in patients with a cervical or thoracic injury. There was no significant difference between patients with DO/DSD and DA in latency of SEP. However, the amplitude of SEP in patients with DA was significantly higher than that of patients with DO/DSD $(\mathrm{P}=0.038)$. Fig. 6D shows that pressure of urine leakage had no association with SEP. However, there was a significant difference in bladder capacity in leakage in patients with and without SEP.

Our results also showed the association of the urodynamic findings with the frequency of the BCR (Fig. 7) $(\mathrm{P}<0.0001)$. There was no significant difference in the latency of BCR and the levels of injury. However, the amplitude of BCR in patients with cervical, thoracic, and lumbar injury was significantly higher than in patients with conical and/or cauda injury.

\section{DISCUSSION}

Our study aimed to conclude the characteristics of detrusor behavior, urodynamic examination findings, and neurophysiological tests in patients with traumatic SCI. It resulted in some important findings: (1) a correlation between level of injury and urodynamic findings but no correlation with degree of injury;
(2) a higher incidence of DBND in patients with cervical injury and those with high thoracic injury; (3) a high incidence of DA in patients with suprasacral injury and normal BCR; (4) a correlation between the duration of injury and urodynamic findings; (5) a relationship of the low $\mathrm{BC}$ and urodynamic findings (detrusor reflexes); (6) a relationship of the level and degree of injury and BS; and (7) a relationship of level of injury and SEP.

Many studies have noted a strong correlation between the level of injury and urodynamic findings but a weak or no correlation between degree of injury and urodynamic findings [1-4], which is in accord with our findings (Fig. 2). Remarkably, 39\% (54) and $63 \%$ (58) of patients with complete and incomplete suprasacral injury, respectively, and a normal BCR response presented with DA. Previous reports have shown that patients with suprasacral injury may experience DA due coexistent clinical or subclinical SCI, but the cases of those reports were relatively small [11-13]. Light et al. [14] reported on 6 patients with suprasacral injury who showed intact somatic sacral reflux on neurophysiological testing. Light et al. [14] thought this unexpected prolonged DA might be related to the dissociation between recovery of the suprasacral and sacral portions of the spinal cord and between the somatic and automatic nervous system. While the somatic nervous system recovers after the spinal shock stage, the autonomic nervous system may not recover. This hypothesis matched our observation that the duration of injury in patients with DO/DSD was significantly longer than in patients with DA at each injury level (Fig. 3). Another possible reason is that bladder overdistension in the shock period may impair impulse conduction through bladder muscle, influencing the bladder reflex [14,15]. However, we also found that incomplete SCI patients with normal BCR had a higher incidence of DA than those with complete injury. Unfortunately, we could not provide a reasonable explanation for this phenomenon.

We noticed DSD in patients with SCI, while neglecting DBND. Our results are similar to those of previous reports in which the incidence pattern of DBND closely followed the incidence pattern of DSD [16,17]. However, the incidence of DBND in patients with a cervical injury (21\%) was similar to that in patients with an upper thoracic injury. The mechanism of DBND is complex. Sympathetic fibers originating in the T11-L2 segments in the spinal cord innervate the bladder neck [18]; thus, impairment above this level might more easily lead to DBND. In addition, the incidence pf DBND in patients with a lumbar injury was higher than that in the cervical injury group in cur- 
rent study, but this might mean little due to the relatively small sample size of the lumbar injury group.

Previous reports have indicated that BC is associated with bladder hyperreflexia or reflexes in patients with lower motor neuron injuries [1], which is in accord with our findings in which 117 patients (34\%) with low BC had DO/DSD, compared with 52 (17\%) in patients with conical cauda injuries. Weld et al demonstrated that low $\mathrm{BC}$ was more common in patients with a sacral injury than in those with a suprasacral injury and more common in patients with a complete injury rather than an incomplete injury for each bladder management type [19], but we did not observe this, which can be explained by the relatively shorter time after injury in our study.

Afferent nerves originate in the bladder and travel via the pelvic, pudendal, and hypogastric nerves to the central nervous system [20-22]. Of these three nerves, hypogastric nerves (T11L2) cause BS in the most proximal spinal level [20]. Theoretically, in patients with complete lesions, especially below T10, the bladder retains some innervations for sensation, which has been reported previously [21,22]. According to Wyndaele [21], 15 of 42 patients (35.7\%) with complete SCI had BS and most of them had a lower thoracic or lumbar lesion. In these patients, BS might be preserved through the hypogastric nerves. Ersoz and Akyuz [22] reported that they observed BS in all patients with incomplete SCI (21 cases) and 82\% (28 of 34 cases) of those with complete lesions below T10. The authors speculated that sensation-dependent bladder emptying develops in many patients with SCI [22]. However, these reports focused on the lower thoracic, lumbar lesion, or conical cauda injury. In our study, preserved BS was more common in patients with incomplete than with complete injury and in patients with lesions below T10 than those with lesions above T10, which is in accord with previous reports. Of all patients, $13.8 \%$ (27 of 195) had a complete injury above T10, which we hypothesize is possibly related to nonspinal sensory pathways because the pelvic organ has dual sensory innervations from spinal and nonspinal (i.e., vagus nerve) sources.

Previous studies have observed qualitative changes in BS in SCI patients, which means that the patients could not accurately feel the first sensation of bladder filling or the first or strong desire to void [22]. We also found these changes qualitatively during urodynamic testing; thus, we categorized BS as absent or preserved. Adequate sensory input is required for conscious bladder control and to store and expel urine at a convenient time and place [21]. Our results also showed that, compared with patients without BS, bladder capacity during urine leakage is far higher in those with BS. We conjecture that BS is helpful for urine storage in patients with SCI.

BCR and SEP are used to evaluate the function of the pudendal nerve. Investigators always doubt the values for BCR and SEP for bladder management in patients after SCI $[7,23]$. Previous studies have noted that DO/DSD is significantly related to normal sacral reflex in patients with SCI, and abnormal or absent sacral reflex was observed in patients with a suprasacral injury $[7,23,24]$. These findings are in accordance with our results. We also found that the amplitude of the BCR was higher in patients with a suprasacral injury, which might be related to the interruption of descending inhibitory pathways from the brain.

Bladder capacity during urine leakage in patients with preserved SEP was significantly higher than in patients with an absent SEP, which is in parallel with BS. One confusing result is that the amplitude of SEP in patients with DU was significantly higher than that in patients with DO/DSD. This might be a meaningless result due to the significant difference in number of patients in the above 2 groups. We believe that the current pelvic floor electrophysiological examination was helpful but may have limited meaning in bladder management for patients with SCI.

The main limitation of this study is this retrospective study could not include SCI patients with normal micturition reflex, because most patients with relative normal voiding did not accept video-urodynamic evaluation or pelvic floor electrophysiological examination because the tests were expensive. Due to these objective conditions, we find little patients with normal micturition reflex in those SCI patients. A prospective study needs to do to evaluate the bladder function of all of traumatic SCI patients. Additionally, hospitalized patients with lower urinary dysfunction induced by SCI were usually treated with intermittent catheterization in our center, but a high proportion of SCI patients were outpatients, they always accepted indwelling catheters. Because the space is limited, we will analysis what differences of urodynamic results between patients with indwelling and with intermittent catheterization in another article.

In conclusion, there is a significant correlation between the level of SCI and video-urodynamic findings, but clinical examination cannot accurately predict bladder function. Urodynamic examination is necessary to determine the function of the lower urinary tract; however, pelvic floor electrophysiological examination do not seem to be essential for SCI patients. Additional- 
ly, BS might be helpful for urinary control in patients with traumatic SCI patients; further study is needed.

\section{SUPPLEMENTARY MATERIAL}

Supplementary Material can be found via https://doi.org/10.5213/ inj.2040376.188.

\section{AUTHOR CONTRIBUTION STATEMENT}

- Conceptualization: $L L$

- Data curation: $Z W, H D, X L$

- Formal analysis: $Z W$

- Methodology: $Z W$

- Project administration: $L L$

-Writing-original draft: $Z W$

-Writing-review \& editing: $Z W$

\section{ORCID}

$\begin{array}{ll}\text { Zhaoxia Wang } & 0000-0003-2767-4956 \\ \text { Han Deng } & 0000-0002-9509-8623 \\ \text { Xing Li } & 0000-0002-9116-8700 \\ \text { Limin Liao } & 0000-0002-7092-6576\end{array}$

\section{REFERENCES}

1. Agrawal M, Joshi M. Urodynamic patterns after traumatic spinal cord injury. J Spinal Cord Med 2015;38:128-33.

2. Weld KJ, Dmochowski RR. Association of level of injury and bladder behavior in patients with post-traumatic spinal cord injury. Urology 2000;55:490-4.

3. Kaplan SA, Chancellor MB, Blaivas JG. Bladder and sphincter behavior in patients with spinal cord lesions. J Urol 1991;146:113-7.

4. Wyndaele JJ. Correlation between clinical neurological data and urodynamic function in spinal cord injured patients. Spinal Cord 1997;35:213-6.

5. Wein AJ. Classification of neurogenic voiding dysfunction. J Urol 1981;125:605-9.

6. Tankisi H, Pugdahl K, Rasmussen MM, Clemmensen D, Rawashdeh YF, Christensen P, et al. Pelvic floor electrophysiology in spinal cord injury. Clin Neurophysiol 2016;127:2319-24.

7. Shenot PJ, Rivas DA, Watanabe T, Chancellor MB. Early predictors of bladder recovery and urodynamics after spinal cord injury. Neurourol Urodyn 2015;17:25-9.
8. Kirshblum SC, Burns SP, Biering-Sorensen F, Donovan W, Graves $\mathrm{DE}$, Jha $\mathrm{A}$, et al. International standards for neurological classification of spinal cord injury (revised 2011). J Spinal Cord Med 2011; 34:535-46.

9. Schäfer W, Abrams P, Liao L, Mattiasson A, Pesce F, Spangberg A, et al. Good urodynamic practices: uroflowmetry, filling cystometry, and pressure-flow studies. Neurourol Urodyn 2002;21:261-74.

10. Abrams P, Cardozo L, Fall M, Griffiths D, Rosier P, Ulmsten U, et al. The standardisation of terminology in lower urinary tract function: report from the standardisation sub-committee of the International Continence Society. Urology 2003;61:37-49.

11. Bradley WE, Conway CJ. Bladder representation in the pontinemesencephalic reticular formation. Exp Neurol 1966;16:237-49.

12. Fletcher TF, Bradley WE: Neuroanatomy of the bladder-urethra. J Urol 1978;119:153-60.

13. Arnold EP, Fukui J, Anthony A, Utley WL. Bladder function following spinal cord injury: a urodynamic analysis of the outcome. Br J Urol 1984;56:172-7.

14. Light JK, Faganel J, Beric A. Detrusor areflexia in suprasacral spinal cord injuries. J Urol 1985;148:295-7.

15. Iwatsubo E, Komine S, Yamashita H, Imamura A, Akatsu T. Overdistension therapy of the bladder in paraplegic patients using selfcatheterisation: a preliminary study. Paraplegia 1984;22:210-5.

16. Krongrad A, Sotolongo JR Jr. Bladder neck dysynergia in spinal cord injury. Am J Phys Med Rehabil 1996;75:204-7.

17. Schurch B, Yasuda K, Rossier AB. Detrusor bladder neck dyssynergia revisited. J Urol 1994;152:2066-70.

18. Fowler CJ, Griffiths D, de Groat WC. The neural control of micturition. Nat Rev Neurosci 2008;9:453-66.

19. Weld KJ, Graney MJ, Dmochowski RR. Differences in bladder compliance with time and associations of bladder management with compliance in spinal cord injured patients. J Urol 2000;163: 1228-33.

20. de Groat WC. Mechanisms underlying the recovery of lower urinary tract function following spinal cord injury. Paraplegia 1995; 33:493-55.

21. Wyndaele JJ. Investigation of the afferent nerves of the lower urinary tract in patients with 'complete' and 'incomplete' spinal cord injury. Paraplegia 1991;29:490-4.

22. Ersoz M, Akyuz M. Bladder-filling sensation in patients with spinal cord injury and the potential for sensation-dependent bladder emptying. Spinal Cord 2004;42:110-6.

23. Koldewijn EL, Van Kerrebroeck PE, Bemelmans BL, Notermans SL, Lemmens WA, Debruyne FM. Use of sacral reflex latency measurements in the evaluation of neural function of spinal cord injury 


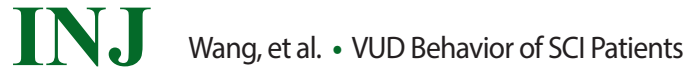

patients: a comparison of neuro-urophysiological testing and urodynamic investigations. J Urol 1994;152(2 Pt 1):463-7.

24. Lucas MG, Thomas DG. Lumbosacral evoked potentials and vesi- courethral function in patients with chronic suprasacral spinal cord injury. J Neurol Neurosurg Psychiatry 1990;53:982-6. 